\title{
EchoGéo
}

\section{Savoir lire les centres historiques comme clé pour construire la ville de demain}

Entretien avec Yves Dauge, Rio de Janeiro, 26/03/10

\section{Alexis Sierra et Karine Peyronnie}

\section{OpenEdition}

\section{Journals}

\section{Édition électronique}

URL : https://journals.openedition.org/echogeo/11896

DOI : $10.4000 /$ echogeo.11896

ISSN : 1963-1197

\section{Éditeur}

Pôle de recherche pour l'organisation et la diffusion de l'information géographique (CNRS UMR 8586)

\section{Référence électronique}

Alexis Sierra et Karine Peyronnie, "Savoir lire les centres historiques comme clé pour construire la ville de demain », EchoGéo [En ligne], 12 | 2010, mis en ligne le 31 mai 2010, consulté le 01 août 2021. URL: http://journals.openedition.org/echogeo/11896 ; DOl : https://doi.org/10.4000/echogeo.11896

Ce document a été généré automatiquement le 1 août 2021.

EchoGéo est mis à disposition selon les termes de la licence Creative Commons Attribution - Pas d'Utilisation Commerciale - Pas de Modification 4.0 International (CC BY-NC-ND) 


\section{Savoir lire les centres historiques comme clé pour construire la ville de demain}

Entretien avec Yves Dauge, Rio de Janeiro, 26/03/10

Alexis Sierra et Karine Peyronnie

1 Yves Dauge est sénateur d'Indre et Loire (membre de la commission de la culture, de l'éducation et de la communication, membre du groupe d'études sur le patrimoine architectural). Inspecteur général de l'équipement, ancien maire de Chinon, il a été délégué interministériel à la ville et au développement social urbain de 1988 à 1991. Il a conduit la délégation française au V ${ }^{\text {ème }}$ Forum Urbain Mondial à Rio. Il anime avec les services du Ministère des Affaires Etrangères et Européennes, le nouveau Partenariat Français pour la Ville et les Territoires qui regroupe tous les acteurs français, publics et privés, de la coopération urbaine.

2 - Alexis Sierra (AS), Karine Peyronnie (KP). Le Forum Urbain Mondial est une rencontre internationale qui porte sur un espace et des territoires spécifiques. À ce titre, il est éminemment politique puisque l'existence de ces territoires urbains implique la présence d'élus et de gouvernements locaux. Pourtant, dans la préparation de ce Forum Urbain Mondial vous êtes le seul parlementaire à vous être fortement impliqué. Il y a bien des élus de certaines villes françaises (Grenoble, Nanterre, Angers) mais au niveau national est-ce que d'après votre expérience, l'urbain et la ville sont portés politiquement?

3 - Yves Dauge (YD). Je ne crois pas que la question urbaine soit portée dans le monde politique.

Internationalement comme nationalement, les approches sont sectorielles. Les Nations Unies sont ce que les Etats veulent; or, ceux-ci portent de grandes thématiques (pauvreté, eau, changement climatique, etc.) qui sont relayées par les Nations Unies. Les Etats font écran à des questions plus territoriales qui elles sont portées par les collectivités territoriales. Ainsi, la question urbaine, comme objet particulier de réflexion au niveau des Nations Unies, n'existe que depuis une quinzaine d'années, 
depuis le forum d'Istanbul, parce qu'il a été porté par les municipalités et les gouvernements locaux.

4 En mettant la question urbaine en avant, en réfléchissant sur les politiques urbaines, il ne s'agit pas de remettre en cause la réflexion sur certaines thématiques mondiales. Il s'agit d'avoir une autre grille de débat et d'inscrire ces grands thèmes dans du territoire, dans de la démocratie, dans de l'action [publique]. L'entrée urbaine permet de traduire de la thématique, qui reste essentielle, dans une dimension territoriale. Cela permet de la transversalité. Un autre thème fédérateur pourrait être les grands fleuves.

La ville soulève la question des pouvoirs locaux, de la gouvernance. Elle peut être un vrai sujet de coopération [internationale]. En France, la problématique urbaine dans la coopération a été plus difficile à mettre en œuvre pour diverses raisons : la France n'a pas de grandes métropoles, est devenue urbaine tardivement, et la colonisation l'a tournée vers les questions agraires et rurales. Les institutions comme l'Agence Française de Développement (AFD) ont été moins équipées intellectuellement pour travailler sur l'urbain et sa complexité que sur la complexité rurale. Ainsi, lorsque je me suis engagé dans une coopération pour la sauvegarde du patrimoine à Luang-Prabang, ancienne capitale royale du Laos, il a été nécessaire de mettre en place des outils adaptés à la réalité du lieu. Pour cela, il a fallu penser la ville autrement : la structure urbaine repose sur un ensemble de villages, structurés autour d'un temple et non sur une structure linéaire le long d'une rue ou d'un axe. La colonisation n'a pas détruit la structure antérieure, mais a apporté une dimension linéaire entre les villages et le fleuve. Travailler sur la question patrimoniale et créer un secteur sauvegardé implique de savoir lire la ville dans cette complexité ce qui n'était pas évident au départ, notamment pour le bailleur de fonds, l'AFD, dont les compétences et les financements se focalisaient auparavant sur les questions d'infrastructures.

6 - AS, KP. Dans votre combat sur l'urbain, avez-vous constaté une forte présence de géographes? Quelle est selon vous leur utilité ? Comment les impliquer davantage dans les politiques sur la ville?

7 - YD. Il y a à la fois des besoins en géographie et une présence peu marquée. Comment faire un plan de cohérence territoriale sans des géographes ? Il faut à la fois penser la question des risques, le paysage, l'architecture, la sauvegarde des espaces et des activités agricoles... Très souvent ces plans sont faits par des ingénieurs et des techniciens. Il n'y a pas de regard sur le paysage, sur l'articulation avec la nature, avec les risques, ... Du côté du ministère de l'écologie, de l'énergie, du développement durable et de la mer (MEDEEM), les approches manquent de lisibilité. Elles se limitent à une technicité, la production de normes, l'idée de "ville économe» en fonction des innovations techniques. Ce faisant, ces acteurs étatiques perdent les fondamentaux de la planification urbaine. La politique de la ville est devenue illisible à tel point qu'un conflit brutal avec les services publics générateurs de violence - maintien de l'ordre dans les quartiers - peut être interprété comme un retour à la colonisation. Le vocabulaire témoigne d'un rapport de haine.

Quid du projet global de ville qui doit être pensé en amont ? Pourtant ce projet peut nous aider à réfléchir et à développer toutes les thématiques portées internationalement (eau, environnement, etc.).

8 Dans ce contexte, le Partenariat pour la Ville et les Territoires que nous mettons en place doit s'ouvrir à des disciplines et des acteurs qui ont pu être exclus de l'élaboration de la politique de la ville : les chercheurs (géographes, spécialistes du risque), les ONG, 
les élus. Pour ce faire, il convient de reconnaitre toutes les compétences dans ces domaines, par exemple dans les bureaux d'études (Gret, Ateliers de Cergy ${ }^{1}$, etc.).

Il y a désormais une course de vitesse, un rapport de force à créer. La volonté politique et l'intelligence sont souvent trop faibles pour faire reculer la spéculation. Il faut donc renforcer l'institutionnel et les compétences. Il faut garder une ligne politique y compris en travaillant avec les acteurs privés pour servir l'intérêt général et lutter contre l'intérêt immédiat.

10 - AS, KP. Le Forum Urbain Mondial, la coopération française dans le domaine des villes, est l'occasion de repenser le développement. Nous avons vu cependant que les villes sont bien différentes y compris au sein des pays dits du Nord comme au sein des pays dits du Sud. A partir de votre expérience, est-ce que la division Nord-Sud vous semble encore pertinente?

11 - YD. C'est difficile à dire. En France, nous pouvons rencontrer des situations très dures de pauvreté, d'exclusion, de violence. Elles sont parfois plus dures que dans des pays du Sud. En Inde, il y a des violences intercommunautaires mais on peut trouver dans les bidonvilles des formes de démocraties approfondies et renouvelées.

12 Dans les "Suds", on va être en avance dans une certaine pratique collective et démocratique. Le Sud est porteur d'innovation d'un savoir-vivre ensemble cependant que le Nord crée des quartiers écologues... Il y a aussi des questions ethniques, des déterminismes urbanistiques et architecturaux qui rendent en apparence plus complexe ce découpage Nord/Sud mais en regardant de près ce n'est pas pertinent.

13 - AS, KP. Au side-event sur les centres historiques ${ }^{2}$, vous avez mis l'accent sur les centres des villes, mais les villes du monde sont différentes, elles ne sont pas toutes construites autour d'un centre ancien comme en Europe. Certaines sont polycentriques par exemple. La vision et la représentation du patrimoine est également très différente d'un pays à l'autre. En Chine, on a beaucoup détruit ce qui est ancien. Quelle vision de la ville peut-on apporter dans un travail de coopération internationale?

14 - YD. La Chine bascule très vite, la rapidité d'action des Chinois est remarquable. Avec le soutien de la Direction de l'architecture et du patrimoine du Ministère de la culture et de la communication français, l'université de Tongji à Shanghai met en place une méthodologie pour la sauvegarde de son centre ancien qui est amenée à être développée dans d'autres villes chinoises. Il faut rappeler qu'en France, il y a quarante ans, on détruisait les centres dans une vision hygiéniste. La défense du patrimoine a commencé par quelques militants qui ont défendu les centres comme à Tours et ensuite les élus ont suivi. Sur la question urbaine, beaucoup de villes dans le monde vont voir que ces quartiers anciens représentent des ressources en termes économiques, de lien social et en termes esthétiques.

15 En Europe, en France il y a de nombreuses expériences sur les transports collectifs, le logement social, la mixité comme moteur de cœur de ville qui donne sens au projet urbain.

16 La politique de la ville prend conscience de la notion de centralité. Il ne s'agit pas de dire qu'il y a un seul centre, ce n'est pas que le centre historique, il peut y avoir plusieurs centres comme une ville en archipel mais l'idée est « halte à la ville qui bouffe de l'espace sans limites »! On détruit des espaces agricoles, on s'installe dans des zones à risque. La ville correspond à des générations de traditions urbaines. Il y a toute une histoire dans la trame urbaine et des relations multiples comme avec l'eau. 


\section{d'autres villes.}

18 - AS, KP. La participation officielle à ce genre de rencontre internationale implique de mettre en valeur un savoir-faire français. N'y a-t-il pas cependant le risque d'une arrogance?

19 - YD. La culture en donne un exemple. Nous devons repositionner le réseau culturel français et rompre avec le concept d'agence culturelle où tout est fourni y compris les artistes français. Les temps ont changé. Notre proposition est d'aller sur le terrain pour produire ensemble avec les résidents, révéler des talents locaux, mettre en place des outils à partir des expériences françaises mais pour le compte des locaux. Cela va en droite ligne de la convention de l'UNESCO sur la diversité culturelle. Il faut parvenir à trouver une alliance avec les forces créatrices locales (théâtre, cinéma, etc.).

En ce qui concerne la ville, on se persuade que les territoires avec lesquels on coopère n'ont aucune ressource mais ils ne nous ont pas attendus! Certes, la France peut apporter des moyens technologiques - comme au théâtre de Ouagadougou au Burkina Faso- mais ces outils doivent être mis à la disposition des acteurs locaux. Ce qui vaut pour l'action culturelle dans ces villes est valable pour les projets urbains. Un investissement intellectuel est nécessaire pour saisir la trame urbaine (histoire coloniale par exemple). Dans certains cas, comme à Luang Prabang, un appui institutionnel peut aussi être apporté.

\section{NOTES}

1. Ces deux bureaux d'études étaient présents à Rio.

2. Table ronde à laquelle participaient l'IRD et l'Unité Mixte de Recherche Prodig à travers Catherine Paquette (chercheur urbaniste).

\section{AUTEURS}

\section{ALEXIS SIERRA}

Alexis Sierra est maître de conférence, géographe et membre de l'UMR PRODIG alexisierra2001@yahoo.fr

\section{KARINE PEYRONNIE}

Karine Peyronnie est chercheur à l'IRD, géographe et membre de l'UMR PRODIG

karine.peyronnie@ird.fr 